\title{
COMMON FIXED POINT THEOREMS FOR NONEXPANSIVE MAPPINGS USING THE LOWER SEMICONTINUITY PROPERTY
}

\author{
SŁAWOMIR BORZDYŃSKI
}

\begin{abstract}
Suppose that $E$ is a Banach space, $\tau$ a topology under which the norm of $E$ becomes $\tau$-lower semicontinuous and $\mathcal{S}$ a commuting family of $\tau$-continuous nonexpansive mappings defined on a $\tau$-compact convex subset $C$ of $E$. It is shown that the set of common fixed points of $\mathcal{S}$ is a nonempty nonexpansive retract of $C$. Along the way, a few other related fixed point theorems are derived.
\end{abstract}

\section{INTRODUCTION}

Let $C$ be a subset of a Banach space $E$. A mapping $T: C \rightarrow C$ is said to be nonexpansive if $\|T x-T y\| \leq\|x-y\|$ for every $x, y \in C$. In this paper we shall study fixed point properties of commutative semigroups of nonexpansive mappings defined on $\tau$-compact convex subsets of a Banach space $E$ with respect to a Hausdorff topology $\tau$ on $E$. For more information on fixed point theory for nonexpansive mappings, confer e.g., [GK] or [GD].

The following fixed point theorem for a commuting family of nonexpansive mappings was proved in [BW].

Theorem 1.1. Suppose that $C$ is a nonempty weak*-compact convex subset of a dual Banach space and $\mathcal{S}$ is an arbitrary family of commuting weak*continuous nonexpansive self-mappings on $C$. Then $\operatorname{Fix} \mathcal{S}$, the set of the common fixed points of the family $\mathcal{S}$, is a nonexpansive retract of $C$.

Recall that a non-void set $D \subset C$ is a nonexpansive retract of $C$ if there exists a nonexpansive mapping $R: C \rightarrow D$ such that the restriction of $R$ to $D$ is the identity. Then $R$ is called a nonexpansive retraction. The above theorem enabled us to confirm a special case of the long-standing open problem originally posed by A. T.-M. Lau. (check [L], [L2] or [LZ, Question 1] for details).

It is known that the norm is lower semicontinuous in the weak* topology. Given a Hausdorff topology $\tau$, the $\tau$-lower semicontinuity of the norm means

2010 Mathematics Subject Classification. Primary 47H10; Secondary 46B20, 47H09.

Key words and phrases. Nonexpansive mapping, Fixed point. 
that

$$
\left\|\tau-\lim _{\alpha} x_{\alpha}\right\| \leq \liminf _{\alpha}\left\|x_{\alpha}\right\|
$$

for any $\tau$-convergent net $\left(x_{\alpha}\right)_{\alpha} \subset E$. The other known examples of such topologies are weak and strong (norm) topologies. It turns out that Theorem 1.1 can be generalized to topologies under which the norm becomes lower semicontinuous. This paper provides such a generalization and-in the process-other related ones. But first, we also show some theorems that follow from Theorem 1.1.

\section{Results}

We start with the statement that bears some resemblance to a remarkable theorem of Bader, Gelander, and Monod [BGM].

Theorem 2.1. Let $\mathcal{S}$ be a commuting family of weak*-continuous nonexpansive mappings defined on a dual Banach space $E$. Let furthermore $\mathcal{S}$ preserve a bounded set $A$ (i.e., $T A=A$ for all $T \in \mathcal{S}$ ). Then there is a common fixed point of $\mathcal{S}$ which is located in $\mathcal{C}(A)$, the Tchebyshev center of $A$.

Before the proof, let us recall the definition of the Tchebyshev center:

$$
\mathcal{C}(A)=\{c \in E: A \subset \mathbb{B}(c, r(A))\},
$$

where $\mathbb{B}$ denotes the closed ball, and $r(A)$ is called the Tchebyshev radius of the set $A$ :

$$
r(A)=\inf \left\{r \geq 0: \exists_{x \in E} A \subset \mathbb{B}(x, r)\right\} .
$$

Proof. In the dual space $E$, it is known that $\mathcal{C}(A)$ is nonempty, bounded, convex and $w^{*}$-compact (cf. e.g., $[\mathrm{BGM}]$ ). So the only thing to do is to show the $\mathcal{S}$-invariance of it, and use Theorem 1.1. Take $T \in \mathcal{S}$. If $r$ denotes the Tchebyshev radius of $A$ and $c \in \mathcal{C}(A)$, then from the fact that $A \subset \mathbb{B}(c, r)$, we can derive

$$
A=T A \subset T \mathbb{B}(c, r) \subset \mathbb{B}(T c, r) .
$$

The latter inclusion is true because if $z \in T \mathbb{B}(c, r)$, then obviously there exists $x \in \mathbb{B}(c, r)$ such that $z=T x$, meaning

$$
\|T c-z\|=\|T c-T x\| \leq\|c-x\| \leq r,
$$

that is, $z \in \mathbb{B}(T c, r)$. Thus we have $A \subset \mathbb{B}(T c, r)$, meaning $T c \in \mathcal{C}(A)$. From the freedom of choice of $c$ and $T$ we conclude that $\mathcal{C}(A)$ is $\mathcal{S}$-invariant, which-as stated before-proves the theorem.

Using the Kuratowski-Zorn lemma, we can derive the following lemma. 
Lemma 2.2. For every commuting family $\mathcal{S}$ of continuous mappings defined on a compact set $C$, there exists a compact subset of $C$ on which every mapping in $\mathcal{S}$ is surjective.

The property from the above lemma was called the subsurjectivity in BW2. Now we can state

Theorem 2.3. Let $\mathcal{S}$ be a commuting family of weak*-continuous nonexpansive mappings defined on a dual Banach space E. Suppose there exists a $w^{*}$-compact, $\mathcal{S}$-invariant set $C \subset E$. Then the family $\mathcal{S}$ has a common fixed point.

Proof. Use lemma 2.2 to obtain a set $B \subset C$ with the same properties as $C$ but on which $\mathcal{S}$ is surjective. Since $B$ is bounded (because of $w^{*}$ compactness), using theorem 2.1yields a common fixed point of $\mathcal{S}$ in $E$.

Note, since the Tchebyshev center property is not monotone $(A \subset B \not \Longrightarrow$ $\mathcal{C}(A) \subset \mathcal{C}(B)$ ), we cannot state this time that a fixed point of $\mathcal{S}$ is located in $\mathcal{C}(C)$. Let us show one more example of an argument that utilizes the concept of subsurjectivity. To this end, we will make use of the following definition.

Definition 2.4. For a family $\mathcal{S}$ of mappings defined on a set $C$, let $\gamma \mathcal{S}$ denote the maximal subset of $C$, on which all elements from the semigroup generated by $\mathcal{S}$ commute. If $\gamma \mathcal{S} \neq \emptyset$, we will say that $\mathcal{S}$ is somewhere commuting (on $C$ ).

Let us list basic properties of the $\gamma$ operation.

Lemma 2.5. The following claims are true:

(1) the set $\gamma \mathcal{S}$ is $\mathcal{S}$-invariant (i.e. $T \in \mathcal{S} \Longrightarrow T(\gamma \mathcal{S}) \subset \gamma \mathcal{S}$ ),

(2) $F i x \mathcal{S} \subset \gamma \mathcal{S}$,

(3) if the mappings from $\mathcal{S}$ are continuous in some topology, then $\gamma \mathcal{S}$ is closed in it.

Proof. Claim (1)

For a fixed $T \in \mathcal{S}$, take $x \in \gamma \mathcal{S}$. Then for any $T_{1}, T_{2} \in \mathcal{S}$,

$$
T_{1} T_{2} T x=T_{1}\left(T_{2} T\right) x=T_{2}\left(T T_{1} x\right)=T_{2} T_{1} T x
$$

and from the maximality of $\gamma \mathcal{S}, T x$ must be also the element of $\gamma \mathcal{S}$. Claim (2)

If $T_{1}, T_{2} \in \mathcal{S}$ and $x \in$ Fix $\mathcal{S}$, then obviously $T_{1} T_{2} x=x=T_{2} T_{1} x$ and $x \in \gamma \mathcal{S}$, by the maximality argument. 
Claim (3)

Take a net $\left(x_{\alpha}\right) \subset \gamma \mathcal{S}$ with $\lim _{\alpha} x_{\alpha}=x$. Then the mappings from $\mathcal{S}$ commute over element $x$ :

$$
T_{1} T_{2} x=T_{1} T_{2} \lim _{\alpha} x_{\alpha}=\lim _{\alpha} T_{1} T_{2} x_{\alpha}=\lim _{\alpha} T_{2} T_{1} x_{\alpha}=T_{2} T_{1} \lim _{\alpha} x_{\alpha}=T_{2} T_{1} x .
$$

And again, we can use the maximality of $\gamma \mathcal{S}$ to infer the desired result.

Note that the above lemma can be stated shortly: if $\mathcal{S}$ is a family of continuous mappings commuting at least at one point, then there is a closed $\mathcal{S}$-invariant superset of Fix $\mathcal{S}$, on which $\mathcal{S}$ commutes.

Lemma 2.6. If $\mathcal{S}$ is a family of continuous mappings on a compact set $C$ which commute at least over one point, then there exists a compact subset of $C$ on which $\mathcal{S}$ consists of commuting surjections.

Proof. Let $\left.\mathcal{S}\right|_{C}=\left\{\left.T\right|_{C}: T \in \mathcal{S}\right\}$. With the aid of Lemma 2.5, we see that $A=\gamma\left(\left.\mathcal{S}\right|_{C}\right)$ is a nonempty, $\mathcal{S}$-invariant, compact set on which $\mathcal{S}$ commutes. Then use Lemma 2.2 to obtain another set $B \subset A$ with the same properties, but on which $\mathcal{S}$ is surjective.

As it is known (cf. [K] and [LN, p. 61] for the proof):

Theorem 2.7 (Freudenthal-Hurewicz). A nonexpansive surjection on a compact metric space is an isometry.

We obtain instantly

Theorem 2.8. If $\mathcal{S}$ is a family of nonexpansive mappings, commuting somewhere (and defined) on the compact metric space $C$, then there exists a compact set $A \subset C$ on which $\mathcal{S}$ are surjective commuting isometries.

Thus $\left.\mathcal{S}\right|_{A}$ generates the abelian group of isometries. Now, let us deal with the generalizations related to Theorem 1.1 .

Theorem 2.9. Let $C$ be a $\tau$-compact subset of a normed linear space $E$, where the norm is $\tau$-lower semicontinuous $(\tau-L S)$ and let $\mathcal{S}$ be a family of $\tau$-continuous mappings on $C$ with the following property: for every finite subfamily $\mathcal{A} \subset \mathcal{S}, F i x \mathcal{A}$ is a nonexpansive retract of $C$. Then Fix $\mathcal{S}$ is also a nonexpansive retract of $C$.

Proof. We may assume that $\mathcal{S}$ is infinite. For later use, notice that for $T \in \mathcal{S}$ the set FixT is $\tau$-closed. Consider

$$
\Lambda=\{\alpha \subset \mathcal{S}: \# \alpha<\infty\}
$$


as the directed set with the order relation $\leq$ as inclusion. Denote by $R_{\alpha}$ a nonexpansive retraction from $C$ to $F i x_{\alpha}=\bigcap_{T \in \alpha} F i x T$, whose existence follows from the assumptions. This construction gives us a net $\left(R_{\alpha}\right)_{\alpha \in \Lambda}$ in the $\tau$-compact space $C^{C}$ (Tychonoff's theorem), and we can select a $\tau$ convergent subnet $\left(R_{\alpha_{\gamma}}\right)_{\gamma \in \Gamma}$. Using the definition of the compact topology, it is easy to deduce that we can define a mapping $R$ such that for every $x \in C$,

$$
R x=\tau-\lim _{\gamma} R_{\alpha_{\gamma}} x
$$

For $T \in \mathcal{S}$, take $\gamma_{0}$ such that for every $\gamma \geq \gamma_{0}$, we have $\alpha_{\gamma} \geq\{T\}$. It follows straightforward from subnet's definition. Then for any $x \in C$,

$$
\forall_{\gamma \geq \gamma_{0}} R_{\alpha_{\gamma}} x \in F i x_{\alpha_{\gamma}} \subset F i x_{\alpha_{\gamma_{0}}} \subset F i x T
$$

and hence, $R_{\alpha_{\gamma}} x$ lies eventually in the $\tau$-closed set FixT. That is, $R x \in$ FixT, and from the freedom of choice of $T$ and $x$, we have $R C \subset F i x \mathcal{S}$. But also

$$
x \in \text { Fix } \mathcal{S} \Longrightarrow x \in F i x_{\alpha} \Longrightarrow R_{\alpha} x=x \Longrightarrow R x=x \Longrightarrow x \in \text { FixR. }
$$

Hence $R C \subset F i x \mathcal{S} \subset F i x R \subset R C$, which shows that $R$ is a retraction from $C$ to $F i x \mathcal{S}$. It remains to use the $\tau$-lower semicontinuity to prove that $R$ is nonexpansive:

$\|R x-R y\|=\left\|\tau-\lim _{\gamma} R_{\alpha_{\gamma}} x-\tau-\lim _{\gamma} R_{\alpha_{\gamma}} y\right\| \leq \liminf _{\gamma}\left\|R_{\alpha_{\gamma}} x-R_{\alpha_{\gamma}} y\right\| \leq\|x-y\|$.

Remark 2.10. The above theorem can be easily modified at least in a few ways:

(1) we can replace the $\tau$-continuity of mappings from $\mathcal{S}$ with an assumption that their fixed point sets are $\tau$-closed,

(2) the proof can be stated in metric spaces, assuming the following definition of $\tau$-LS

$$
d\left(\tau-\lim _{\alpha} x_{\alpha}, 0\right) \leq \liminf _{\alpha} d\left(x_{\alpha}, 0\right),
$$

(3) let $\mathcal{S}$ be a family of continuous mappings defined on a compact set $C$. If for every finite subfamily $\mathcal{A} \subset \mathcal{S}$ there exists a retraction $C \rightarrow F i x \mathcal{A}$, then the same holds for the whole family $\mathcal{S}$,

(4) Recall the firmly nonexpansive mapping $T: C \rightarrow C$ is defined by the following equation

$$
\|T x-T y\| \leq\|a(x-y)+(1-a)(T x-T y)\|
$$


, which needs to hold for every $a \in(0,1)$. Then notice that if $\tau$ denotes the strong topology and every $F i x \mathcal{A}$ is a firmly nonexpansive retract, then FixS is also a firmly nonexpansive retract- rewrite equation (2.1) from the end of the proof (with $a \in(0,1)$ ):

$$
\begin{gathered}
\|R x-R y\|=\left\|\lim _{\gamma} R_{\alpha_{\gamma}} x-\lim _{\gamma} R_{\alpha_{\gamma}} y\right\|= \\
\lim _{\gamma}\left\|R_{\alpha_{\gamma}} x-R_{\alpha_{\gamma}} y\right\| \leq \lim _{\gamma}\left\|a(x-y)+(1-a)\left(R_{\alpha_{\gamma}} x-R_{\alpha_{\gamma}} y\right)\right\|= \\
\left\|a(x-y)+(1-a)\left(\lim _{\gamma} R_{\alpha_{\gamma}} x-\lim _{\gamma} R_{\alpha_{\gamma}} y\right)\right\|=\|a(x-y)+(1-a)(R x-R y)\|,
\end{gathered}
$$

(5) we can change a 'nonexpansive retract' to a ' $\tau$-continuous affine retract' (i.e. retract, for which there exists a $\tau$-continuous affine retraction) in the assumptions. As a result, we get an affine retract this time. The $\tau$-LS assumption is superfluous. Again, we only need to modify the end of the original proof.

As we can see, Theorem (2.9) may have many variants but in order to fuel them, the finite case theorem is always needed. Otherwise, they may be vacuously true. One source of such supporting theorems is the method used by Bruck [B], and recently by Saedi [SM]. To extract it in a general way, for the readability, let us provide the following definition.

Definition 2.11. For a given family $\mathcal{S}$ of mappings defined on a set $C$, let $\mathcal{R}(\mathcal{S})$ denote the set of all retractions from $C$ to $F i x \mathcal{S}$. For the singleton we use the shorthand $\mathcal{R}(\{T\})=\mathcal{R}(T)$.

Since we will use it later, we give without proof the following simple lemma (cf. [BW]).

Lemma 2.12. Let $\mathcal{S}$ be a family of mappings and suppose there exists a retraction $R$ onto Fix $\mathcal{S}$. If $T$ commutes with every member of the family $\mathcal{S}$, then FixT $\cap$ Fix $\mathcal{S}=$ FixTR.

Theorem 2.13. Let a semigroup $\mathcal{A} \subset C^{C}$ has the following property:

$$
\forall_{T \in \mathcal{A}} \mathcal{R}(T) \cap \mathcal{A} \neq \emptyset .
$$

Then for every finite commuting family $\mathcal{S} \subset \mathcal{A}$ we have also

$$
\mathcal{R}(\mathcal{S}) \cap \mathcal{A} \neq \emptyset \text {. }
$$

Proof. If family $\mathcal{S}$ is a singleton, then theorem is true directly from the assumptions. Let the theorem be true for the families with $n$ elements. Consider $\mathcal{S}_{n+1}=\left\{T_{1}, \ldots, T_{n+1}\right\}$ and it's subfamily $\mathcal{S}_{n}=\left\{T_{1}, \ldots, T_{n}\right\}$. Then from the induction hypothesis exists retraction $R_{n} \in \mathcal{R}\left(\mathcal{S}_{n}\right) \cap \mathcal{A}$. Since 
$T_{n+1} R_{n} \in \mathcal{A}$, there is also in $\mathcal{A}$ a retraction $R_{n+1}$ from $C$ to $\operatorname{Fix}\left(T_{n+1} R_{n}\right)$. Lemma 2.12 gives Fix $\left(T_{n+1} R_{n}\right)=F i x \mathcal{S}_{n+1}$, so $R_{n+1}$ belongs also to $\mathcal{R}\left(\mathcal{S}_{n+1}\right)$. This proves the theorem via mathematical induction.

The idea is that $\mathcal{A}$ represents a well behaved property of mappings, e.g. nonexpansivity, isometricity, being affine, and so on. Nevertheless, the presented reasoning would fail if the set of interest from Theorem 2.13-the family $\mathcal{A}$-would not posses the semigroup structure or, more specifically, we could not know whether $T_{n+1} R_{n} \in \mathcal{A}$. In those cases we must look for the alternate ways of generating the appropriate retraction $R_{n+1}$. One of such arguments will be presented below.

Lemma 2.14. Let $\mathcal{S}$ be a commuting family of nonexpansive mappings and suppose there exists a nonexpansive retraction $R$ onto FixS. Suppose that a nonexpansive $T$ commutes with members of $\mathcal{S}$. Then every approximate fixed point sequence $\left(x_{n}\right)$ of $T R$ is also the approximate fixed point sequence of both the family $\mathcal{S}$ and the mapping $T$.

Proof. Note the following fact: if $\left(x_{n}\right)$ is an approximate fixed point sequence of the mapping $Q$, and for a nonexpansive $P$ we have $P Q x_{n}=Q x_{n}$, then $\left(x_{n}\right)$ is also an approximate fixed point sequence of $P$. Indeed

$$
\begin{gathered}
\left\|P x_{n}-x_{n}\right\| \leq\left\|P x_{n}-Q x_{n}\right\|+\left\|Q x_{n}-x_{n}\right\|= \\
\left\|P x_{n}-P Q x_{n}\right\|+\left\|Q x_{n}-x_{n}\right\| \leq 2\left\|Q x_{n}-x_{n}\right\| \rightarrow 0 .
\end{gathered}
$$

Now, notice that for any $S \in \mathcal{S}$ from the lemma we have

$$
S T R x_{n}=T S R x_{n}=T R x_{n}
$$

so if we denote $Q=T R$ and $P=S$, we obtain $\left\|S x_{n}-x_{n}\right\| \rightarrow 0$. From the equation (2.3) we also conclude that $T R x_{n}$ is a common fixed point of the family $\mathcal{S}$, which gives $R T R x_{n}=T R x_{n}$. Now, letting $Q=T R$ and $P=R$, yields $\left\|R x_{n}-x_{n}\right\| \rightarrow 0$. It follows that

$$
\left\|T x_{n}-x_{n}\right\| \leq\left\|T x_{n}-T R x_{n}\right\|+\left\|T R x_{n}-x_{n}\right\| \rightarrow 0 .
$$

Theorem 2.15. Let $C$ be a nonempty $\tau$-compact convex and bounded subset of a Banach space such that its norm is $\tau-L S$. Let $\mathcal{S}$ be a finite commuting family of nonexpansive $\tau$-continuous self mappings on $C$. Then Fix $\mathcal{S}$ is a nonempty nonexpansive retract of $C$.

Proof. Let us impose one more restriction on $\mathcal{S}$ : it has to possess identity as an element. It is easy to see, that if we would have proof for such families, then the theorem would also follow for the unrestricted ones. So let's 
continue with the restricted families. Then if $\mathcal{S}$ is a singleton, the theorem is trivially true: the retraction we are looking for is just the identity on $C$.

In the spirit of the mathematical induction, let us now assume that there exists a nonexpansive retraction for the given $\mathcal{S}_{n}=\left\{T_{1}, \ldots, T_{n}\right\}$ :

$$
R_{n}: C \rightarrow \text { Fix } \mathcal{S}_{n}
$$

From that we are going to construct another nonexpansive retraction (for the family $\left.\mathcal{S}_{n+1}=\mathcal{S}_{n} \cup\left\{T_{n+1}\right\}\right)$ :

$$
R_{n+1}: C \rightarrow F i x \mathcal{S}_{n+1}
$$

which proves our point.

Note that $C$ is strongly closed: assume $x_{\alpha} \rightarrow x$ strongly. From $\tau$-compactness of $C$, take a subnet $\left(x_{\alpha_{\gamma}}\right)_{\gamma}$ that has a $\tau$-limit in $C$. Then

$$
\left\|\tau-\lim _{\gamma} x_{\alpha_{\gamma}}-x\right\| \leq \liminf _{\gamma}\left\|x_{\alpha_{\gamma}}-x\right\|=\lim _{\gamma}\left\|x_{\alpha_{\gamma}}-x\right\|=0 .
$$

That is, $x=\tau$ - $\lim _{\gamma} x_{\alpha_{\gamma}} \in C$. So $C$ is strongly closed, thus complete. Take

$$
T_{x, s} z=\frac{1}{s} x+\left(1-\frac{1}{s}\right) T_{n+1} R_{n} z .
$$

Since $T_{x, s}$ is a contraction defined on the complete space, there exists exactly one point $F_{s} x \in C$ such that $T_{x, s} F_{s} x=F_{s} x$. This defines the mapping $F_{s}: C \rightarrow C$. Note that

$$
\begin{aligned}
\left\|F_{s} x-F_{s} y\right\|= & \left\|\frac{1}{s}(x-y)+\left(1-\frac{1}{s}\right)\left(T_{n+1} R_{n} F_{s} x-T_{n+1} R_{n} F_{s} x\right)\right\| \leq \\
& \frac{1}{s}\|x-y\|+\left(1-\frac{1}{s}\right)\left\|F_{s} x-F_{s} y\right\|
\end{aligned}
$$

from which we deduce the nonexpansivity of $F_{s}$. Notice further that the fact

$$
F_{s} x=T_{x, s} F_{s} x=\frac{1}{s} x+\left(1-\frac{1}{s}\right) T_{n+1} R_{n} F_{s} x
$$

and boundedness of $C$ implies

$$
\left\|T_{n+1} R_{n} F_{s} x-F_{s} x\right\|=\frac{1}{s}\left\|T_{n+1} R_{n} F_{s} x-x\right\| \leq \frac{\operatorname{diam} C}{s} \rightarrow 0,
$$

that is, $\left(F_{s}\right)_{s \in \mathbb{N}} \subset C^{C}$ forms with every $x \in C$ an approximate fixed point sequence of the mapping $T_{n+1} R_{n}$. From Lemma 2.14 we conclude that the same is true for the family $\mathcal{S}_{n+1}$. Since $C$ is $\tau$-compact, then-from Tychonoff's theorem- $\left(F_{s}\right)_{s \in \mathbb{N}}$ has a convergent subnet (in a pointwise convergence with respect to $\tau$-topology):

$$
R_{n+1}=\tau-\lim _{\alpha} F_{s_{\alpha}}
$$

Then, for every $T \in \mathcal{S}_{n+1}$,

$\left\|T R_{n+1} x-R_{n+1} x\right\|=\left\|T\left(\tau-\lim _{\alpha} F_{s_{\alpha}} x\right)-\tau-\lim _{\alpha} F_{s_{\alpha}}\right\| x \leq \liminf _{\alpha}\left\|T F_{s_{\alpha}} x-F_{s_{\alpha}} x\right\| \rightarrow 0$, 
where convergence is the mentioned consequence of Lemma 2.14, This gives $T R_{n+1} x=R_{n+1} x$, and from the freedom of choice of $x$ and $T$ we have $R_{n+1} C \subset F i x S_{n+1}$. Observe that

$$
x \in F i x T_{n+1} R_{n} \Longrightarrow T_{x, s} x=x \Longrightarrow F_{s} x=x \Longrightarrow R_{n+1} x=x
$$

which means $F i x T_{n+1} R_{n} \subset F i x R_{n+1}$. From Lemma 2.12, follows

$$
F i x R_{n+1} \subset R_{n+1} C \subset F i x \mathcal{S}_{n+1} \subset F i x T_{n+1} R_{n} \subset F i x R_{n+1},
$$

from which we see $R_{n+1} C=F i x \mathcal{S}_{n+1}=F i x R_{n+1}$. So, $R_{n+1}$ is a retraction onto $F i x \mathcal{S}_{n+1}$. Its nonexpansivness follows from the fact that $F_{s}$ is nonexpansive for every $s$, and from $\tau$-LS property (check the eq. (2.1)).

Now, having in mind Theorem 2.9, we have obviously the following result.

Theorem 2.16. Let $C$ be a $\tau$-compact, convex and bounded subset of a $B a$ nach space with $\tau$-LS norm. Then the set of common fixed points of a commuting family of $\tau$-continuous nonexpansive mappings on $C$ is a nonempty nonexpansive retract of $C$.

Notice that we can infer also (compare [B2, Theorem 3])

Theorem 2.17. Let $C$ be a nonempty compact convex subset of a Banach space. Then the fixed point set of a commuting family of nonexpansive mappings on $C$ is a nonempty firmly nonexpansive retract of $C$.

Proof. It is known that the mappings $F_{s}$ defined in the proof of Theorem 2.15 are firmly nonexpansive(cf. GK, Theorem 11.3]). It means that also their strong limit would be firmly nonexpansive. In effect, the 'strong topology-version' of Theorem 2.15 gives us firmly nonexpansive retracts. Then it suffices to use Remark 2.10, (44) to finish the proof.

\section{REFERENCES}

[BGM] U. Bader, T. Gelander, N. Monod, A fixed point theorem for $L_{1}$ spaces, Invent. Math. 189 (2012), 143-148.

[B] R. E. Bruck, Jr., A common fixed point theorem for a commuting family of nonexpansive mappings, Pacific J. Math. 53 (1974), 59-71.

[B2] R. E. Bruck, Nonexpansive projections on subsets of Banach spaces, Pacific J. Math. Volume 47, Number 2 (1973), 341-355.

[BW] S. Borzdyński, A.Wiśnicki, A common fixed point theorem for a commuting family of weak* continuous nonexpansive mappings, Studia Math. 225 (2014), 173-181. 
[BW2] S. Borzdyński, A.Wiśnicki, Applications of uniform asymptotic regularity to fixed point theorems, J. Fixed Point Theory Appl. 18 (2016), $855-866$.

[GK] K. Goebel, W. A. Kirk, Topics in Metric Fixed Point Theory, Cambridge University Press, Cambridge, 1990.

[GD] A. Granas, J. Dugundji, Fixed Point Theory, Springer-Verlag, New York, 2003.

[K] W. A. Kirk, Metric fixed point theory: old problems and new directions, Fixed Point Theory 11(2010), 45-58.

[L] A. T.-M. Lau, Some fixed point theorems and their applications to $\mathrm{W}^{*}$-algebras, Fixed Point Theory and Applications, S. Swaminathan (ed.), Academic Press, New York, 1976, 121-129.

[L2] A.T.-M. Lau, Amenability and fixed point property for semigroup of nonexpansive mappings, M.A. Thera, J.B. Baillon (Eds.), Fixed Point Theory and Applications, Pitman Res. Notes Math. Ser., vol. 252, Longman Sci. Tech., Harlow (1991), pp. 303-313

[LZ] A.T.-M. Lau, Y. Zhang, Fixed point properties for semigroups of nonlinear mappings and amenability. J. Funct. Anal. 263 (2012), 29492977.

[LN] B. Lemmens, R. Nussbaum, Nonlinear Perron-Frobenius Theory, Cambridge University Press, 2012

[SM] S. Saeidi, I. Mohamadi, Existence and structure of the common fixed points based on tvs, Faculty of Sciences and Mathematics, Filomat 31, No 6 (2017), 1773-1779.

SŁawomir Borzdyński, Institute of Mathematics, Maria Curie-SkŁodowska University, 20-031 Lublin, Poland

E-mail address: slawomir.borzdynski@gmail.com 